\title{
Intercellular transfer of messenger RNAs in multiorgan tumorigenesis by tumor cell-derived exosomes
}

\author{
HONG JIANG ${ }^{1}$, ZHENG LI $^{2}$, XIAOHUA LI ${ }^{1}$ and JIANGUO XIA ${ }^{1}$ \\ ${ }^{1}$ Department of Gastrointestinal Surgery, First Affiliated Hospital of Nanjing Medical University, Nanjing, Jiangsu 210029, \\ P.R. China; ${ }^{2}$ Department of Food Science and Human Nutrition, Institute of Food and Agricultural Sciences, \\ University of Florida, Gainesville, FL 32611, USA
}

Received February 21, 2014; Accepted November 7, 2014

DOI: $10.3892 / \mathrm{mmr} .2015 .3312$

\begin{abstract}
Exosomes are small membrane vesicles of endocytic origin. They are derived from various cells, including tumor cells, and may serve as important modulators of intercellular communication. The present study established a U-87 MG human glioblastoma cell line that showed a stable expression of green fluorescent protein (GFP; U-87-GFP). Two types of human tumor cell lines, U-87-GFP and LoVo human colon cancer cells, were demonstrated to communicate through the transfer of GFP messenger (m)RNA by exosomes. Furthermore, GFP mRNAs delivered by the U-87-GFP cell-derived exosomes were translated into functional proteins in the recipient LoVo cells. In addition, LoVo cells were demonstrated to uptake exosomes derived from the U-87-GFP cells by clathrin-mediated endocytosis. These results indicate that tumor cell-derived exosomes may represent vesicular carriers that regulate gene expression, which may provide a pathway of intercellular communication in the tumor microenvironment during multiorgan tumorigenesis. The exosome uptake pathway may have potential therapeutic applications in multiorgan tumorigenesis.
\end{abstract}

\section{Introduction}

Exosomes are small $(50-100 \mathrm{~nm})$ membrane vesicles of endocytic origin that are released into the extracellular environment upon fusion of multivesicular bodies $(100 \mathrm{~nm}-1 \mu \mathrm{m})$ with the plasma membrane $(1,2)$. Various cells have been shown to release exosomes, including reticulocytes (3), as well as dendritic (4), B- (5) and T- (6), mast (7), epithelial (8) and

Correspondence to: Dr Jianguo Xia, Department of Gastrointestinal Surgery, First Affiliated Hospital of Nanjing Medical University, 300 Guangzhou Road, Nanjing, Jiangsu 210029, P.R. China

E-mail: jianguo.xia@yahoo.com

Key words: exosomes, intercellular communication, messenger RNA delivery, tumor microenvironment, colon cancer, glioblastoma, multiorgan tumorigenesis, cellular uptake pathway tumor cells (9). In addition, exosomes have been isolated and characterized from various biological fluids, such as urine (10), serum (11) and human breast milk (12). The biological functions of exosomes depend mainly on their surface proteins and the cell types from which they are derived. Although the exosome function remains poorly understood, previous studies have suggested that exosomes may be associated with a wide range of biological processes, such as intercellular communication (13) and the development of immune tolerance (14). Exosomes have been shown to target specific recipient cells, exchanging proteins and lipids between the cells and delivering messenger (m)RNAs and microRNAs (7). Therefore, the exosome-mediated transfer of mRNAs and microRNAs may be a potential mechanism for the exchange of genetic material between cells.

Multiorgan tumorigenesis has low incidence; however, it is associated with poor clinical prognosis. Previous studies have hypothesized that environmental or chemical factors contribute towards multiorgan tumorigenesis (15-17). However, the precise underlying mechanisms of the communication of multiorgan tumors with host stromal cells and tumor cells remain to be elucidated.

The present study evaluated the intercellular communication through exosomes in multiorgan tumorigenesis, using LoVo human colon cancer and U-87 MG human glioblastoma cells. A U-87-green fluorescent protein (GFP) cell line was initially established, which expressed GFP in U-87 MG cells. U-87-GFP cell-derived exosomes were hypothesized to transfer GFP mRNAs, which would then be translated into proteins in the recipient LoVo cells. The present study aimed to reveal the function of exosomes in intercellular communication during multiorgan tumorigenesis. The cellular uptake pathway of U-87-GFP cell-derived exosomes into LoVo cells was also investigated in order to reveal how exosomes are associated with intercellular communication between cancer cells, which may have potential therapeutic applications.

\section{Materials and methods}

Cell lines. The LoVo human colon cancer and U-87 MG ATCC ${ }^{\circledast}$ HTB-14 ${ }^{\mathrm{TM}}$ human glioma cell lines were obtained from the American Type Culture Collection (Manassas, VA, USA). U-87-GFP cells expressing GFP were generated by 
stable infection of U-87 MG cells with a lentiviral vector expressing GFP (pHAGE-CMV-MCS-IZs green vector; provided by Professor Chun Lu, Nanjing Medical University, Nanjing, China). The LoVo cells were grown in RPMI-1640 medium supplemented with $10 \%$ fetal bovine serum, $100 \mathrm{U} / \mathrm{ml}$ penicillin and $100 \mu \mathrm{g} / \mathrm{ml}$ streptomycin (all Life Technologies, Grand Island, NY, USA). The U-87-GFP cells were grown in Dulbecco's modified Eagle's medium supplemented with $10 \%$ fetal bovine serum, $100 \mathrm{U} / \mathrm{ml}$ penicillin and $100 \mu \mathrm{g} / \mathrm{ml}$ streptomycin (Life Technologies). All the cells were maintained at $37^{\circ} \mathrm{C}$ in a humidified atmosphere containing $5 \% \mathrm{CO}_{2}$.

Isolation of exosomes. The U-87-GFP cells were cultured in microvesicle-free medium generated by ultracentrifugation in an SW41 rotor (Beckman Instruments, Fullerton, CA, USA) at $100,000 \mathrm{x} \mathrm{g}$ for $1 \mathrm{~h}$ and the culture medium $\left(2 \times 10^{7}\right.$ cells $)$ was collected following a $48 \mathrm{~h}$ incubation. The U-87-GFP cell-derived exosomes were purified by a series of differential centrifugations, as previously described (18). Briefly, the collected culture supernatants were centrifuged for $10 \mathrm{~min}$ at $300 \mathrm{x} g$ in order to eliminate cell contamination. Subsequently, the supernatants were further centrifuged at $1,200 \mathrm{x} g$ for $20 \mathrm{~min}$ and then at $10,000 \mathrm{x}$ g for $30 \mathrm{~min}$. The supernatant from the final spin was ultracentrifuged (Beckman SW-41; Beckman Coulter, Inc., Brea, CA, USA) at 100,000 x g for $2 \mathrm{~h}$ in order to pellet the exosomes. Next, the exosome pellet was resuspended in $200 \mu \mathrm{l}$ phosphate-buffered saline (PBS) and filtered through a $0.22 \mu \mathrm{m}$ filter (EMD Millipore, Bedford, MA, USA). The protein concentration of exosomes was assessed using a Quick Start ${ }^{\mathrm{TM}}$ Bradford Protein assay (Bio-Rad Laboratories, Inc., Hercules, CA, USA).

Characteristics of exosomes. In order to determine the exosome morphology, the U-87-GFP cell-derived exosome dispersion $(10 \mu \mathrm{l})$ was loaded onto a Formvar/carbon coated grid (Agar Scientific, Stansted, UK) for $1 \mathrm{~min}$, negatively stained with $10 \mu \mathrm{l}$ neutral $1 \%$ aqueous phosphotungstic acid (Sigma-Aldrich, St. Louis, MO, USA) for $1 \mathrm{~min}$ and visualized using an electron microscope (XL-30 ESEM FEG; FEI, Hillsboro, OR, USA). The mean particle size (number-weighted size distribution) and $\xi$-potential of the U-87-GFP cell-derived exosomes were measured by dynamic light scattering using a Zetasizer 3000 (Malvern Instruments, Ltd., Malvern, UK). Briefly, $10 \mu \mathrm{l}$ of the exosomes was dispersed into $1 \mathrm{ml}$ deionized water. The resultant dispersion was analyzed in triplicate and each replicate was analyzed eleven times in order to yield the average particle size. Similarly, the $\xi$-potential of each dispersion sample was investigated in triplicate and each replicate was analyzed eleven times to obtain the average $\zeta$-potential.

Quantitative polymerase chain reaction $(q P C R)$ and reverse transcription-PCR (RT-PCR). The cells or exosomes were harvested and total RNA was extracted using the TRIzol ${ }^{\circledR}$ reagent (Invitrogen Life Technologies, Carlsbad, CA, USA). In total, $2 \mu \mathrm{g}$ total RNA was isolated, according to the manufacturer's instructions, and cDNA was synthesized using a conventional method (SuperScript ${ }^{\circledR}$ III; Life Technologies). qPCR was performed using SYBR ${ }^{\circledR}$ Green PCR Master mix (Applied Biosystems Life Technologies, Foster City, CA, USA) on an ABI 7300 Prism real-time PCR (Applied Biosystems Life
Technologies) instrument. The following primers were used for qPCR amplification in the present study: GFP sense, 5'-CCA CTGCCATTCTCCGAAGA-3', and antisense, 5'-TGCTGG ATGAAGTGCCTGTC-3'; and $\beta$-actin sense, 5'-AGGGAA ATCGTGCGTGAC-3', and antisense, 5'-CGCTCATTGCCG ATAGTG-3'. The cycle threshold $(\mathrm{Ct})$ values were measured and the $2^{-\Delta \mathrm{Ct}}$ method (where $\Delta \mathrm{Ct}=$ target gene $\mathrm{Ct}-\beta$-actin $\mathrm{Ct}$ ) was used for quantification. The primer sequences used for RT-PCR amplification in the present study were as follows: GFP sense, 5'-AAGTTCATCTGCACCACCG-3', and antisense, 5'-TCCTTGAAGAAGATGGTGCG-3'; and $\beta$-actin sense, 5-TGACGGGGTCACCCACACTGTGCCCATCTA-3', and antisense, 5'-CTAGAAGCATTTGCGGTGGACGAT GGAGGG-3'. All primers were synthesized by GenePharma Co., Ltd (Shanghai, China). In total, 30 cycles of PCR were performed and the annealing temperature was set at $56^{\circ} \mathrm{C}$. The PCR cycle consisted of an initial step of $95^{\circ} \mathrm{C}$ for $3 \mathrm{~min}$, followed by 30 cycles of $95^{\circ} \mathrm{C}$ for $15 \mathrm{sec}$ and $56^{\circ} \mathrm{C}$ for $30 \mathrm{sec}$, followed by an elongation step at $72^{\circ} \mathrm{C}$ for $7 \mathrm{~min}$. The PCR products were confirmed by agarose gel electrophoresis, followed by ethidium bromide staining (Sigma-Aldrich).

Transfer experiments. The LoVo cells $\left(5 \times 10^{4}\right.$ cells) were seeded on 24-well plates prior to incubation. The exosomes $(20 \mu \mathrm{g} / \mathrm{ml})$ were added to the LoVo cells at a ratio of 8:1 between the donor and recipient cells, according to a previously described method (7). Next, the cells were analyzed by fluorescence microscopy (CKX41-URFLT50; Olympus Corporation, Tokyo, Japan), following 0, 24 and $48 \mathrm{~h}$ of incubation.

Cellular uptake of exosomes. U-87-GFP cell-derived exosomes were initially labeled with the PKH26 fluorescent dye (Sigma-Aldrich), according to the manufacturer's instruction. Cellular uptake of U-87-GFP cell-derived exosomes by LoVo cells was conducted by incubating the PKH26-labeled exosomes with the LoVo cells for $12 \mathrm{~h}$, in the presence of $5 \mu \mathrm{M}$ cytochalasin D, $500 \mathrm{nM}$ bafilomycin $\mathrm{A} 1$ or $50 \mu \mathrm{m}$ indomethacin (Sigma-Aldrich). Following the 12-h incubation, the LoVo cells were washed three times with PBS and detached by trypsinization (Life Technologies). The cells were then centrifuged at $300 \mathrm{xg}$ for $5 \mathrm{~min}$ and resuspended in $0.4 \%(\mathrm{w} / \mathrm{v})$ trypan blue (Sigma-Aldrich) in Hank's balanced salt solution (Life Technologies) to quench the extracellular fluorescence. The treated samples were washed and analyzed using a BD FACSCanto $^{\mathrm{TM}}$ system (BD Biosciences, Franklin Lakes, NJ, USA) with 5,000-10,000 cells measured for each sample.

Statistical analyses. Statistical analyses were performed by Student's t-test using SPSS version 16.0 software (SPSS Inc., Chicago, IL, USA). $\mathrm{P}<0.05$ was considered to indicate a statistically significant difference.

\section{Results}

Isolation and identification of tumor cell-derived exosomes. Previous studies have hypothesized that environmental or chemical factors may contribute to multiorgan tumorigenesis $(17,19)$. However, the precise mechanisms underlying the communication of multiorgan tumors with host cells and tumor cells remain to be elucidated. 


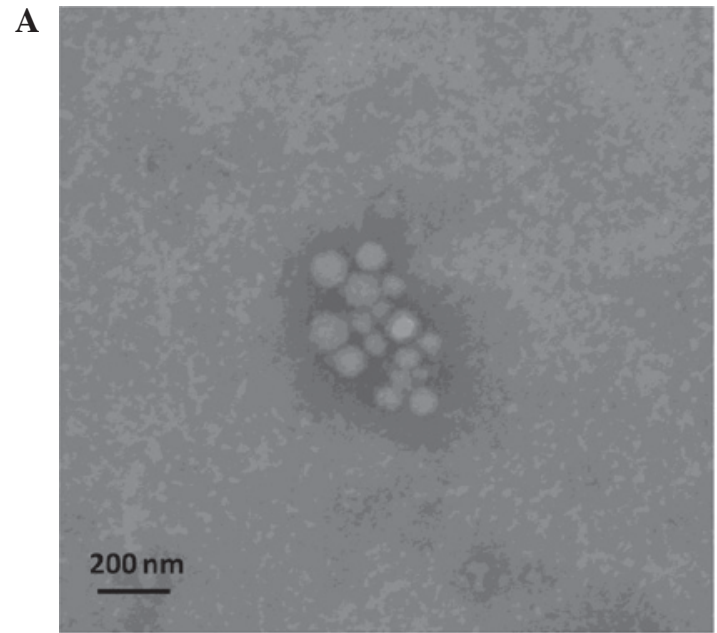

B

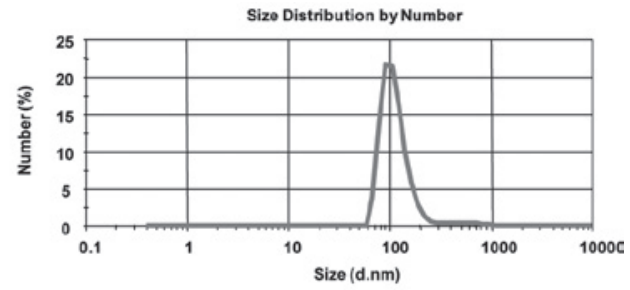

$\mathbf{C}$

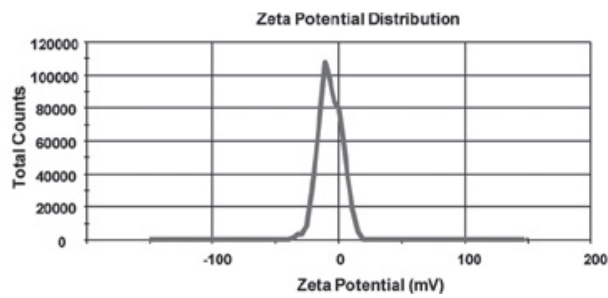

Figure 1. U-87-GFP cells were found to release exosomes, which were isolated and purified. (A) Exosomes are vesicles with sizes of 50-100 nm and were visualized using an electron microscope. (B) Particle size distribution (number-weighted size distribution) of exosomes derived from U-87-GFP cells. (C) $\zeta-P o t e n t i a l$ distribution of exosomes derived from U-87-GFP cells. GFP, green fluorescent protein; U-87-GFP, U-87 MG glioma cells expressing GFP.

A

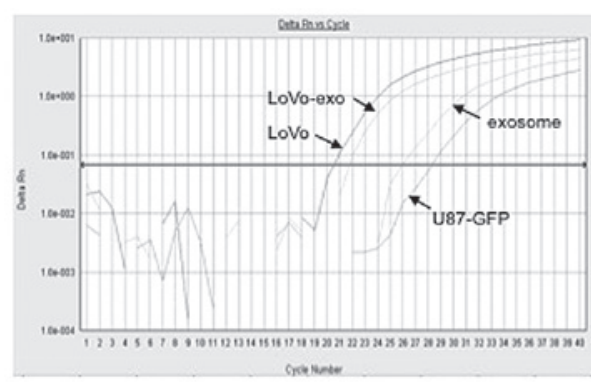

C

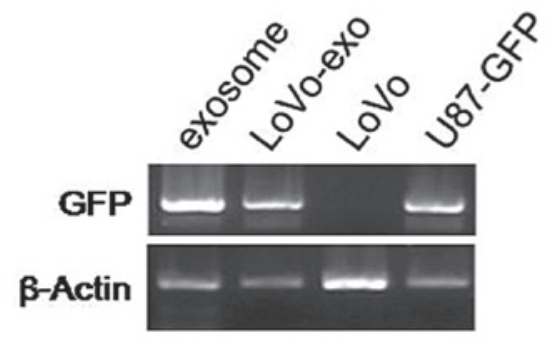

B

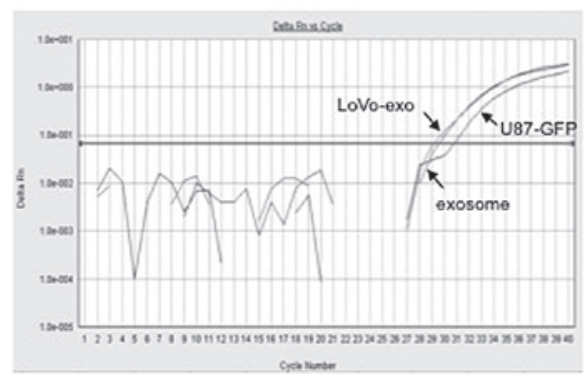

D

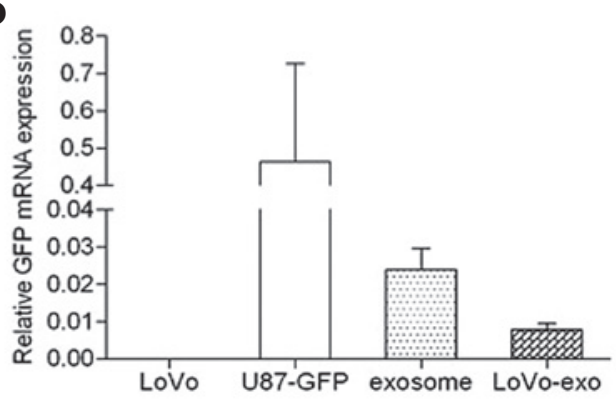

Figure 2. Exosomes derived from U-87-GFP cells were found to contain and deliver GFP mRNAs. Amplification curves of (A) $\beta$-actin and (B) GFP mRNA mRNA, in LoVo cells, U-87-GFP cells, exosomes and LoVo cells incubated with U-87-GFP cell-derived exosomes (LoVo-exo) cells. (C) The transfer of GFP mRNAs was detected using reverse transcription-polymerase chain reaction (RT-PCR). (D) Quantitative PCR was used to detect GFP mRNAs in LoVo cells, U-87-GFP cells, exosomes and LoVo-exo cells. The cycle threshold $(\mathrm{Ct})$ values were measured and the $2^{-\Delta \mathrm{Ct}}$ equation $(\Delta \mathrm{Ct}=\operatorname{target}$ gene $\mathrm{Ct}-\beta$-actin $\mathrm{Ct})$ was used for quantification. The data are presented as the mean \pm standard deviation of three independent experiments. GFP, green fluorescent protein; U-87-GFP, U-87 MG glioma cells expressing GFP.

In the present study, exosomes derived from tumor cells were hypothesized to promote multiorgan tumorigenesis. In order to assess this hypothesis, the most aggressive tumor models were selected, including U-87 MG human glioblastoma and LoVo colon cancer cell lines. Initially, the ability of these tumor cells to produce exosomes was investigated. Next, the U-87-GFP human glioma cell line, showing a stable expression of GFP, was generated. U-87-GFP cell-derived exosomes were isolated from these cells through a series of differential microfiltration and ultracentrifugation steps, modified from a previous study (20). The exosomes were collected and characterized using electron microscopy and dynamic light scattering techniques. The exosomes were constitutively derived from tumor cells (with a density ranging between 0.75 and $1.10 \mathrm{~g} / \mathrm{ml}$ ). Electron micrographs revealed that the exosomes appeared to be round and well-delimited vesicles, with a size of $\sim 100 \mathrm{~nm}$ (Fig. 1A), which was similar to previously described exosomes $(7,20-22)$. Following redispersion of the U-87-GFP cell-derived exosomes in deionized water, the average size and $\zeta$-potential were found to be $140 \mathrm{~nm}$ and $-6.23 \mathrm{mV}$, respectively (Fig. 1B and C). The exosome size observed using dynamic light scattering was comparable with the results obtained using electron microscopy. 


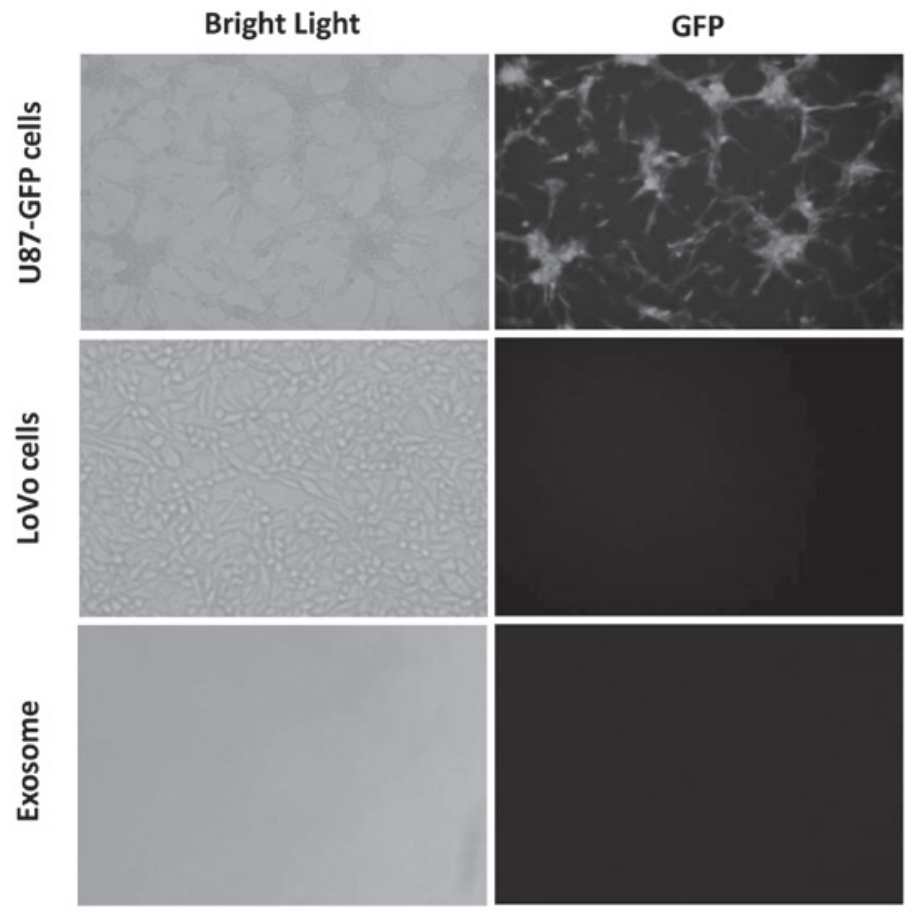

Figure 3. U-87-GFP cells were generated using a lentiviral vector expressing GFP. Fluorescence microscopy detected no GFP fluorescence in LoVo cells and U-87-GFP cell-derived exosomes (magnification, x10). GFP, green fluorescent protein; U-87-GFP, U-87 MG glioma cells expressing GFP.

Intercellular transfer of GFP mRNAs by tumor cell-derived exosomes. The ability of tumor cell-derived exosomes to deliver mRNAs into tumors from different organs/sites was investigated as a potential pathway of intercellular communication during multiorgan tumorigenesis. To determine whether the exosomes were capable of transferring exogenous GFP mRNAs, LoVo cells were incubated with exosomes derived from U-87-GFP cells, which showed a stable expression of GFP. Following incubation with the exosomes for $24 \mathrm{~h}$, GFP mRNA expression was detected using qPCR and was further verified by agarose gel electrophoresis. Amplification of the housekeeping gene $\beta$-actin mRNA expression was detected in the LoVo and U-87-GFP cells, U-87-GFP cell-derived exosomes and LoVo cells incubated with U-87-GFP cell-derived exosomes after 21, 28.2, 25.9 and 22.0 cycles, respectively (Fig. 2A). Notably, no amplification of GFP mRNA expression was detected in the LoVo cells. By contrast, amplification of GFP mRNA was observed in the U-87-GFP cells, U-87-GFP cell-derived exosomes and LoVo cells incubated with U-87-GFP cell-derived exosomes after 31.8, 29.5 and 29.1 cycles, respectively (Fig. 2B). The mRNA expression levels of GFP were highest in the U-87-GFP cells, followed by the U-87-GFP cell-derived exosomes and LoVo cells incubated with U-87-GFP cell-derived exosomes (Fig. 2C). The results obtained from the agarose gel electrophoresis further supported the observation that GFP mRNA was detected in LoVo cells following incubation with U-87-GFP cell-derived exosomes. These data indicated that GFP mRNA was transferred from the U-87-GFP cells to the LoVo cells by U-87-GFP cell-derived exosomes.

GFP mRNAs transferred by exosomes are translated into functional proteins. To determine whether the mRNA transferred by the U-87-GFP cell-derived exosomes was functional, GFP
mRNA delivered by exosomes were investigated to establish whether they can be translated into proteins in the LoVo cells. The exosomes were analyzed by immunofluorescence microscopy (Fig. 3). GFP was detected in the U-87-GFP cells; however, it was not detected in U87-GFP cell-derived exosomes or LoVo cells indicating that they did not contain GFP. Subsequently, LoVo cells were incubated with the exosomes derived from the U-87-GFP cells, and these cells were visualized using immunofluorescence microscopy at the following time points: 0,24 and $48 \mathrm{~h}$. A marked increase in GFP fluorescence was detected in the cytoplasm and near the plasma membrane at $24 \mathrm{~h}$ (Fig. 4). Thus, the GFP mRNA delivered by the exosomes into the LoVo cells was successfully translated into GFP.

Cellular uptake mechanism of exosomes. To clearly identify the role of specific endocytic pathways involved in U-87-GFP cell-derived exosome cellular internalization, LoVo cells were treated with known endocytic inhibitors (Fig. 5). Internalization of U-87-GFP cell-derived exosomes was significantly decreased in the presence of cytochalasin D (29.25\%), an inhibitor of actin polymerization. Clathrin- and caveolae-mediated pathways have been previously shown to require actin polymerization, suggesting that the cellular uptake of U-87-GFP cell-derived exosomes is predominantly achieved through these pathways (23). Furthermore, internalization of the U-87-GFP cell-derived exosomes into LoVo cells was markedly reduced in the presence of bafilomycin A1, a vacuolar proton ATPase inhibitor (49.11\%). However, no inhibitory effect was observed on the cellular uptake of U-87-GFP cell-derived exosomes by LoVo cells in the presence of indomethacin, a caveolae-mediated endocytic inhibitor that blocks the cyclooxygenase pathway $(23,24)$. These results indicated that exosomes derived from U-87-GFP cells were internalized 


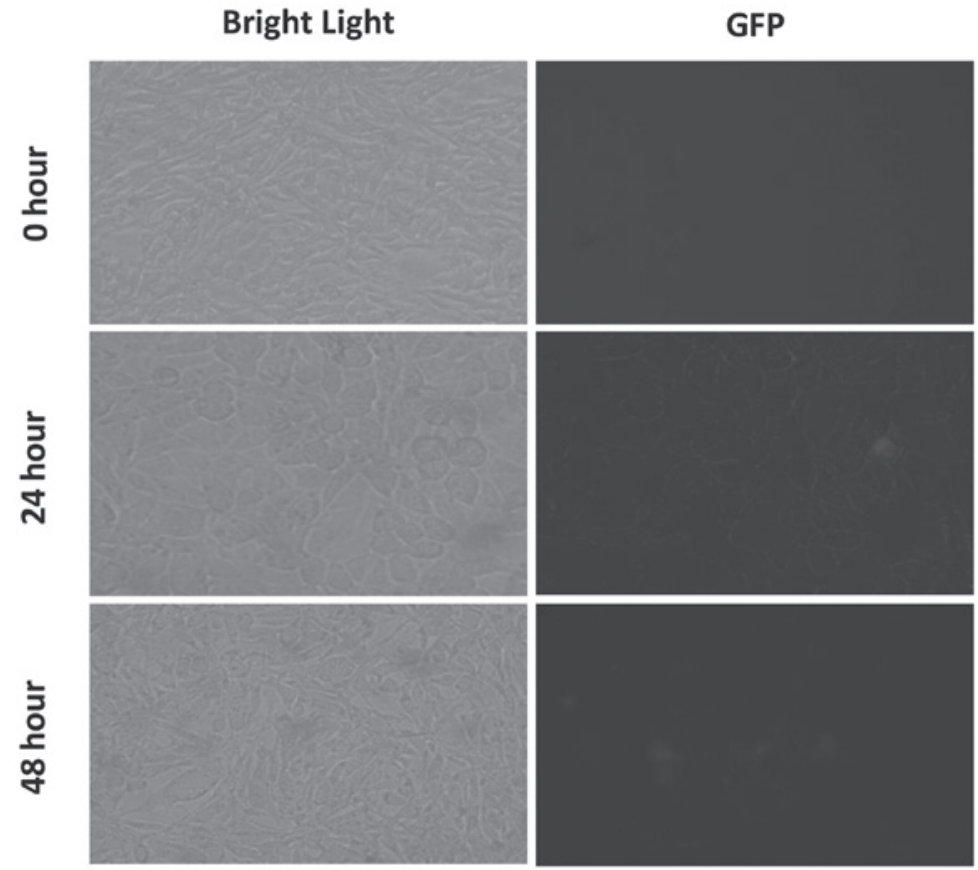

Figure 4. Detection of GFP fluorescence in viable LoVo colon cancer cells incubated with exosomes, derived from U-87-GFP cells, for various incubation periods $(0,24$ and $48 \mathrm{~h}$; magnification, x40). GFP, green fluorescent protein; U-87-GFP, U-87 MG glioma cells expressing GFP.
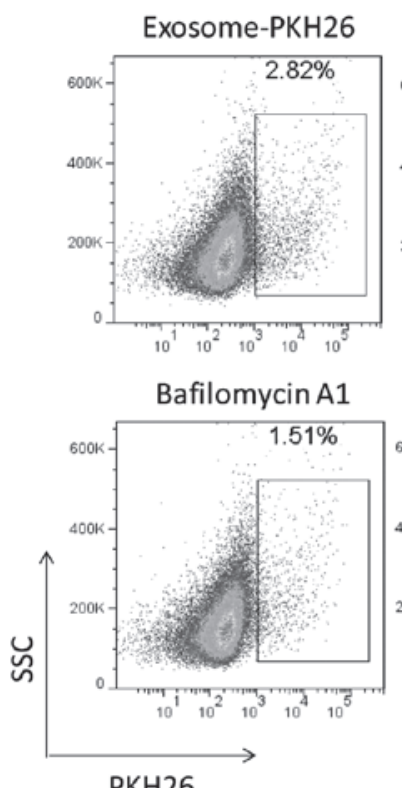

PKH26
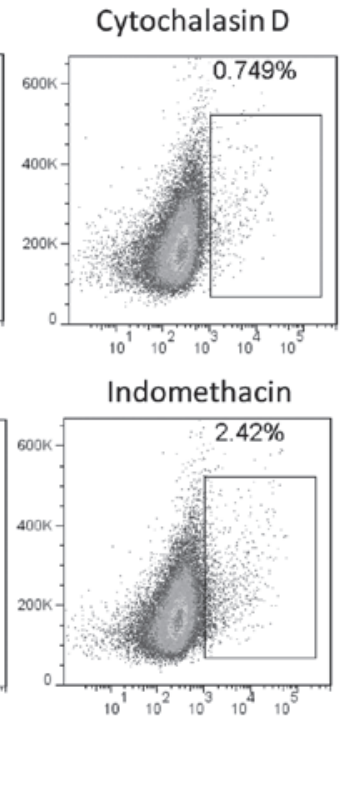

Figure 5. Fluorescence-activated cell sorting analyses of the cellular uptake of PKH26 labeled exosomes, derived from U-87-GFP cells, by LoVo colon cancer cells following a 12-h incubation period in the presence of endocytic inhibitors. The data are presented as the mean \pm standard deviation of three independent experiments. " $\mathrm{P}<0.05 ;{ }^{* *} \mathrm{P}<0.01$ vs. exosome only. GFP, green fluorescent protein; U-87-GFP, U-87 MG glioma cells expressing GFP.

into LoVo cells predominantly through clathrin-mediated endocytosis.

\section{Discussion}

The present study identified a pathway of mRNA transport between different tumor cells by tumor cell-derived exosomes. The tumor cell-derived exosomes were shown to be capable of transferring mRNAs to other types of tumor cells through clathrin-mediated endocytosis. Furthermore, GFP mRNA delivered by exosomes may be translated into protein in the recipient cells. These results indicate that tumor cell-derived exosomes may be capable of delivering mRNAs to recipient cells, which may be a potential pathway of intercellular communication during multiorgan tumorigenesis.

Previous studies have hypothesized that tumor cell-derived microvesicles contain mRNAs that may be transferred (21); however, little is currently known about the precise mechanisms underlying the transfer of RNAs between different tumor types by tumor cell-derived exosomes. A previous 
study indicated that mast cells secrete exosomes that contain mRNAs and microRNAs (7). Based on these previous findings, the present study hypothesized that exosomal RNAs may be transported between tumors from different organs/sites by tumor cell-derived exosomes. The results of the present study demonstrated that exosomes were spontaneously and constitutively derived from tumor cells, which was consistent with the observations of previous studies $(7,20,21,25)$. In addition, these tumor cell-derived exosomes were found capable of transferring GFP mRNA between tumor cells, which may be translated into functional proteins in the recipient cells. Recipient cells were found to uptake exosomes through clathrin-mediated endocytosis. The exosome uptake pathway may have a therapeutic application in multiorgan tumorigenesis.

A previous study has demonstrated that embryonic stem cell microvesicles may be engineered to transfer exogenously-expressed mRNAs and microRNAs (26). In addition, the authors demonstrated that GFP was readily detected in embryonic stem cell microvesicles, where it was less abundant than in embryonic stem cells. By contrast, in the present study, GFP was not detected in the U-87-GFP cell-derived exosomes, indicating that protein transfer by exosomes occurs in a cell type-dependent manner. Simons et al (27) initially proposed the term microvesicles or microparticles for vesicles of heterogeneous size and shape $(100 \mathrm{~nm}-1 \mu \mathrm{m})$ and the term exosomes for vesicles with a size of 40-100 nm that carry the typical exosomal marker proteins. The constituents of microvesicles or exosomes require further investigation, in order to elucidate whether they contain the same constituents. Notably, the results of the present study demonstrated that translation of the mRNA delivered by exosomes was initiated at $24 \mathrm{~h}$ and decreased at $48 \mathrm{~h}$. Therefore, certain mRNAs that are transferred into recipient cells may fall into decay. Future studies should be conducted to examine the mechanisms underlying the regulation of exosome-transferred mRNAs by recipient cells. Based on the present findings, tumor-derived exosomes were hypothesized to deliver genetic information to multiorgan tumors during intercellular communication.

The tumor microenvironment is known to be involved in cancer development and progression. Increasing evidence has indicated that microvesicles/exosomes may be important constituents of the tumor microenvironment, indicating a pivotal role of microvesicles/exosomes in tumor invasion and metastasis, angiogenesis and immune system suppression $(9,28,29)$. Furthermore, previous studies have identified microvesicles/exosomes as major mediators of intercellular communication $(25,30)$. Attention is currently focused on the transfer of nucleic acids by microvesicles/exosomes in intercellular communication. For instance, embryonic stem cell-derived microvesicles have been shown to reprogram hematopoietic progenitors by horizontal transfer of pluripotent and early hematopoietic stem cell mRNA (31). In addition, numerous studies have indicated that microvesicles derived from tumor cells may be involved in the transfer of mRNAs and proteins to antigen-presenting cells (21), as well as oncogenetic receptors among tumor cells (20). Further evidence regarding nucleic acid transfer was provided by a study identifying that mast cell-derived exosomes contain mRNAs and microRNAs, which can be delivered to other cells; these RNAs were termed as 'exosomal shuttle RNAs' (7). In later studies, this novel mechanism of nucleic acid transfer has been observed in tumor cells. Skog et al (18) suggested that glioblastoma microvesicles promote tumor growth through the transportation of RNAs and proteins. Furthermore, Hunter et al (32) identified 33 significantly expressed microRNAs in plasma microvesicles and predicted that these microRNAs may be important factors in the regulation of immune responses and hematopoiesis. In addition, Taylor et al (33) demonstrated that exosomal microRNA profiles from ovarian cancer patients were significantly distinct from the profiles observed in benign diseases. Based on these previous findings, the present study postulated that nucleic acids transferred by tumor cell-derived exosomes, particularly mRNAs and microRNAs, may enable malignant cells to influence the surrounding nonmalignant cells and microenvironment, assisting the tumor in invasion, metastasis and angiogenesis, as well as immune suppression. Furthermore, tumor cells from various organs or sites may communicate by transferring genetic information through tumor cell-derived exosomes, thereby promoting tumor progression during multiorgan tumorigenesis. Inhibition of the uptake pathways of tumor exosomes may have potential therapeutic applications in multiorgan tumorigenesis.

In conclusion, the present study demonstrated that U-87-GFP cell-derived exosomes were capable of transferring mRNAs to LoVo cells, through clathrin-mediated endocytic uptake. The mRNA delivered by the exosomes may then be translated into functional proteins in the recipient LoVo cells. These results indicate that tumor cell-derived exosomes may represent vesicular carriers that regulate gene expression, which may provide a pathway of intercellular communication between tumor cells in the tumor microenvironment during multiorgan tumorigenesis.

\section{References}

1. L Zitvogel, A Regnault, A Lozier, et al: Eradication of established murine tumors using a novel cell-free vaccine: dendritic cell derived exosomes. Nat Med 4: 594-600, 1998.

2. C Théry, L Zitvogel and S Amigorena: Exosomes: composition, biogenesis and function. Nat Rev Immunol 2: 569-579, 2002.

3. Johnstone RM, Bianchini A and Teng K: Reticulocyte maturation and exosome release: transferrin receptor containing exosomes shows multiple plasma membrane functions. Blood 74: 1844-1851, 1989.

4. Théry C, Boussac M, Véron P, et al: Proteomic analysis of dendritic cell-derived exosomes: a secreted subcellular compartment distinct from apoptotic vesicles. J Immunol 166: 7309-7318, 2001.

5. Clayton A, Turkes A, Dewitt S, Steadman R, Mason MD and Hallett MB: Adhesion and signaling by B cell-derived exosomes: the role of integrins. FASEB J 18: 977-979, 2004

6. Blanchard N, Lankar D, Faure F, et al: TCR activation of human T cells induces the production of exosomes bearing the TCR/CD3/zeta complex. J Immunol 168: 3235-3241, 2002.

7. Valadi H, Ekström K, Bossios A, Sjöstrand M, Lee JJ and Lötvall JO: Exosome-mediated transfer of mRNAs and microRNAs is a novel mechanism of genetic exchange between cells. Nat Cell Biol 9: 654-659, 2007.

8. Van Niel G, Mallegol J, Bevilacqua C, et al: Intestinal epithelial exosomes carry MHC class II/peptides able to inform the immune system in mice. Gut 52: 1690-1697, 2003.

9. Wolfers J, Lozier A, Raposo G, et al: Tumor-derived exosomes are a source of shared tumor rejection antigens for CTL cross-priming. Nat Med 7: 297-303, 2001.

10. Nilsson J, Skog J, Nordstrand A, et al: Prostate cancer-derived urine exosomes: a novel approach to biomarkers for prostate cancer. Br J Cancer 100: 1603-1607, 2009. 
11. Caby MP, Lankar D, Vincendeau-Scherrer C, Raposo G and Bonnerot C: Exosomal-like vesicles are present in human blood plasma. Int Immunol 17: 879-887, 2005.

12. Admyre C, Johansson SM, Qazi KR, et al: Exosomes with immune modulatory features are present in human breast milk. J Immunol 179: 1969-1978, 2007.

13. André F, Chaput N, Schartz NE, et al: Exosomes as potent cell-free peptide-based vaccine. I. Dendritic cell-derived exosomes transfer functional MHC class I/peptide complexes to dendritic cells. J Immunol 172: 2126-2136, 2004.

14. Karlsson M, Lundin S, Dahlgren U, Kahu H, Pettersson I and Telemo E: 'Tolerosomes' are produced by intestinal epithelial cells. Eur J Immunol 31: 2892-2900, 2001.

15. Stoner G, Casto B, Ralston S, Roebuck B, Pereira C and Bailey G: Development of a multi-organ rat model for evaluating chemopreventive agents: efficacy of indole-3-carbinol. Carcinogenesis 23 265-272, 2002

16. Pazzaglia S: Ptc1 heterozygous knockout mice as a model of multi-organ tumorigenesis. Cancer Lett 234: 124-134, 2006.

17. Yamagishi M, Natsume M, Osakabe N, et al: Chemoprevention of lung carcinogenesis by cacao liquor proanthocyanidins in a male rat multi-organ carcinogenesis model. Cancer Lett 191: 49-57, 2003.

18. Skog J, Würdinger T, van Rijn S, et al: Glioblastoma microvesicles transport RNA and proteins that promote tumour growth and provide diagnostic biomarkers. Nat Cell Biol 10: 1470-1476, 2008

19. ML Fero, M Rivkin, M Tasch, et al: A Syndrome of multiorgan hyperplasia with features of gigantism, tumorigenesis, and female sterility in p27(Kip1)-deficient mice. Cell 85: 733-744, 1996.

20. Al-Nedawi K, Meehan B, Micallef J, et al: Intercellular transfer of the oncogenic receptor EGFRvIII by microvesicles derived from tumour cells. Nat Cell Biol 10: 619-624, 2008.

21. Baj-Krzyworzeka M, Szatanek R, Weglarczyk K, et al: Tumour-derived microvesicles carry several surface determinants and mRNA of tumour cells and transfer some of these determinants to monocytes. Cancer Immunol Immunother 55: 808-818, 2006.
22. Martinez MC, Larbret F, Zobairi F, et al: Transfer of differentiation signal by membrane microvesicles harboring hedgehog morphogens. Blood 108: 3012-3020, 2006.

23. Gratton SEA, Ropp PA, Pohlhaus PD, et al: The effect of particle design on cellular internalization pathways. Proc Natl Acad Sci USA 105: 11613-11618, 2008.

24. Weggen S, Eriksen JL, Das P, et al: A subset of NSAIDs lower amyloidogenic Abeta42 independently of cyclooxygenase activity. Nature 414: 212-216, 2001

25. Ratajczak J, Wysoczynski M, Hayek F, Janowska-Wieczorek A and Ratajczak MZ: Membrane-derived microvesicles: important and underappreciated mediators of cell-to-cell communication. Leukemia 20: 1487-1495, 2006

26. Yuan A, Farber EL, Rapoport AL, et al: Transfer of microRNAs by embryonic stem cell microvesicles. PLoS One 4: e4722, 2009.

27. Simons M and Raposo G: Exosomes - vesicular carriers for intercellular communication. Curr Opin Cell Biol 21: 575-581, 2009.

28. Janowska-Wieczorek A, Wysoczynski M, Kijowski J, et al: Microvesicles derived from activated platelets induce metastasis and angiogenesis in lung cancer. Int J Cancer 113: 752-760, 2005.

29. Liu C, Yu S, Zinn K, et al: Murine mammary carcinoma exosomes promote tumor growth by suppression of NK cell function. J Immunol 176: 1375-1385, 2006.

30. Schorey JS and Bhatnagar S: Exosome function: from tumor immunology to pathogen biology. Traffic 9: 871-881, 2008.

31. Ratajczak J, Miekus K, Kucia M, et al: Embryonic stem cell-derived microvesicles reprogram hematopoietic progenitors: evidence for horizontal transfer of mRNA and protein delivery. Leukemia 20: 847-856, 2006.

32. Hunter MP, Ismail N, Zhang X, et al: Detection of microRNA expression in human peripheral blood microvesicles. PLoS One 3: e3694, 2008

33. Taylor DD and Gercel-Taylor C: MicroRNA signatures of tumor-derived exosomes as diagnostic biomarkers of ovarian cancer. Gynecol Oncol 110: 13-21, 2008. 